International Journal of Modern Physics E

(C) World Scientific Publishing Company

\title{
Collective neutrino oscillations and spontaneous symmetry breaking
}

\author{
Huaiyu Duan \\ Department of Physics and Astronomy, University of New Mexico \\ Albuquerque, NM 87131, USA \\ duan@unm.edu
}

\begin{abstract}
Neutrino oscillations in a hot and dense astrophysical environment such as a core-collapse supernova pose a challenging, seven-dimensional flavor transport problem. To make the problem even more difficult (and interesting), neutrinos can experience collective oscillations through nonlinear refraction in the dense neutrino medium in this environment. Significant progress has been made in the last decade towards the understanding of collective neutrino oscillations in various simplified neutrino gas models with imposed symmetries and reduced dimensions. However, a series of recent studies seem to have "reset" this progress by showing that these models may not be compatible with collective neutrino oscillations because the latter can break the symmetries spontaneously if they are not imposed. We review some of the key concepts of collective neutrino oscillations by using a few simple toy models. We also elucidate the breaking of spatial and directional symmetries in these models because of collective oscillations.
\end{abstract}

Keywords: collective neutrino oscillations; spontaneous symmetry breaking; core-collapse supernova.

PACS numbers: 14.60.Pq, 97.60.Bw

\section{Introduction}

Neutrinos are abundantly produced in hot and dense astrophysical environments such as the early universe, core-collapse supernovae ( $\mathrm{SNe}$ ) and black-hole accretion discs in which they are also instrumental in the dynamical, thermal and chemical evolution of these environments. In a SN, for example, the hot proto-neutron star (PNS) at the center quickly cools down by emitting $\sim 10^{58}$ neutrinos in all flavors within just $\sim 10$ seconds 11 Outside the PNS electron neutrinos and antineutrinos influence the supernova dynamics and nucleosynthesis through reactions

$$
\nu_{e}+n \leftrightharpoons p+e^{-}, \quad \bar{\nu}_{e}+p \leftrightharpoons n+e^{+} .
$$

Because the weak-interaction states $\left|\nu_{\beta}\right\rangle(\beta=e, \mu, \tau)$ and the mass eigen states $\left|\nu_{i}\right\rangle(i=1,2,3)$ of the neutrino do not coincide with each other, neutrinos can mutate from one flavor (or weak-interaction state) into another during propagation, which is known as the neutrino flavor transformation or neutrino oscillations. ${ }^{2}$ The oscillation of neutrinos between the electron flavor and other flavors can have important physical consequences in astrophysical environments through the reactions in Eq. (1). 
Even in the coherent regime (i.e. without neutrino absorption, emission and collision) the transport of neutrino flavors poses a very challenging problem. One of the challenges is that, in an environment with a large neutrino flux, the flavor transport can become nonlinear because of the neutrino-neutrino coupling. In most cases the nonlinear equations of motion (e.o.m.) which govern neutrino oscillations have to be solved numerically. The computational investigations by various groups show that a dense neutrino medium can experience collective oscillations during which neutrinos of different momenta oscillate cooperatively ${ }^{316}$ To obtain theoretical insights into these numerical results, several toy models such as the bipolar mode ${ }^{17}[19$ have been proposed which can be solved analytically and which bear some of the qualitative features of the more realistic models. Linear stability analysis provides another useful tool to predict the physical regimes where collective neutrino oscillations may or may not occur.20

Another challenge in solving the problem of neutrino transport is simply due to its large dimensionality: the neutrino transport in any real astrophysical environment even without flavor oscillations involves 1 temporal dimension, 3 spatial dimensions and 3 momentum dimensions. This challenge has been largely bypassed in the previous studies which are limited to the models with imposed symmetries. For example, the isotropic and homogeneous condition is usually assumed for the early universe. However, even though such symmetries can exist in the e.o.m. and may also exist in the physical systems at some point, they may not be preserved during neutrino oscillations. ${ }^{21}$ Indeed, a series of recent work suggest that these symmetries can be broken spontaneously by neutrino oscillations.22 27

The goals of this paper are to review some of the key concepts of collective neutrino oscillations through the bipolar neutrino gas model and to explain why spatial and directional symmetries can be broken spontaneously by collective oscillations. We will leave out many important topics such as three-flavor oscillations $11|28| 29$ some of which can be found in an earlier review $\underline{30}$

Throughout this paper we adopt the natural units with $\hbar=c=1$. We also assume that neutrinos are relativistic with speed $v \approx 1$.

\section{Collective neutrino oscillations}

\subsection{Equation of motion}

Assuming the validity of the mean-field theory, $31[32$ we will use the (Wignertransformed) neutrino (flavor) density matrices ${ }^{33} \rho_{\mathbf{p}}(t, \mathbf{x})$ and $\bar{\rho}_{\mathbf{p}}(t, \mathbf{x})$ to describe the flavor content of the neutrino and antineutrino of momentum $\mathbf{p}$ at time $t$ and position $\mathbf{x}$. For simplicity we will consider neutrino oscillations between the electron flavor and another active flavor, say $\tau$. In the weak-interaction basis (or flavor basis),

$$
\rho_{\mathbf{p}}=\left[\begin{array}{cc}
\rho_{\mathbf{p}}^{e e} & \rho_{\mathbf{p}}^{e \tau} \\
\left(\rho_{\mathbf{p}}^{e \tau}\right)^{*} & \rho_{\mathbf{p}}^{\tau \tau}
\end{array}\right], \quad \quad \bar{\rho}_{\mathbf{p}}=\left[\begin{array}{cc}
\bar{\rho}_{\mathbf{p}}^{e e} & \bar{\rho}_{\mathbf{p}}^{e \tau} \\
\left(\bar{\rho}_{\mathbf{p}}^{e \tau}\right)^{*} & \bar{\rho}_{\mathbf{p}}^{\tau \tau}
\end{array}\right]
$$


The diagonal elements of the density matrices in the above equation are proportional to the number densities of the neutrino or antineutrino in the corresponding flavors and momentum state, and

$$
n_{\nu_{\beta}}(t, \mathbf{x})=\int \frac{\mathrm{d}^{3} p}{(2 \pi)^{3}} \rho_{\mathbf{p}}^{\beta \beta}(t, \mathbf{x}), \quad n_{\bar{\nu}_{\beta}}(t, \mathbf{x})=\int \frac{\mathrm{d}^{3} p}{(2 \pi)^{3}} \bar{\rho}_{\mathbf{p}}^{\beta \beta}(t, \mathbf{x})
$$

are the total number densities of the neutrinos and antineutrinos in flavor $\beta$ ( $\beta=$ $e, \tau)$, respectively. The off-diagonal elements of the density matrices contain the information of flavor mixing. In the rest of the paper we will use the weak-interaction basis exclusively unless otherwise stated.

In the coherent regime a neutrino can change its flavor through refraction when it passes through a dense medium. In the lowest-order and without gravitational redshift, the e.o.m. that govern the neutrino flavor transformation $\operatorname{arc}^{\sqrt[33]{35}}$

$$
\begin{aligned}
& \left(\partial_{t}+\hat{\mathbf{v}} \cdot \nabla\right) \rho_{\mathbf{p}}=-\mathrm{i}\left[\mathrm{H}_{\mathrm{vac}}+\mathrm{H}_{\mathrm{mat}}+\mathrm{H}_{\nu \nu}, \rho_{\mathbf{p}}\right], \\
& \left(\partial_{t}+\hat{\mathbf{v}} \cdot \nabla\right) \bar{\rho}_{\mathbf{p}}=-\mathrm{i}\left[-\mathrm{H}_{\mathrm{vac}}+\mathrm{H}_{\mathrm{mat}}+\mathrm{H}_{\nu \nu}, \bar{\rho}_{\mathbf{p}}\right],
\end{aligned}
$$

where $\hat{\mathbf{v}}=\mathbf{p} / E$ is the propagation velocity of the neutrino with $E=|\mathbf{p}|$ being the energy of the neutrino. The vacuum Hamiltonian is

$$
\mathrm{H}_{\mathrm{vac}}=\frac{\Delta m^{2}}{4 E}\left[\begin{array}{cc}
-\cos 2 \theta_{\mathrm{v}} & \sin 2 \theta_{\mathrm{v}} \\
\sin 2 \theta_{\mathrm{v}} & \cos 2 \theta_{\mathrm{v}}
\end{array}\right],
$$

where $\Delta m^{2}$ is the neutrino mass-squared difference, and $\theta_{\mathrm{v}}$ is the vacuum mixing angle within range $(0, \pi / 4]$. Here $\Delta m^{2}>0$ and $\Delta m^{2}<0$ correspond to the normal neutrino mass hierarchy $(\mathrm{NH})$ and the inverted hierarchy $(\mathrm{IH})$, respectively. The matter potential in Eq. (4) arises from the coherent forward scattering of the neutrino by the charged leptons in the medium through the charged-current weak interaction. ${ }^{[36}$ When the densities of $\mu$ and $\tau$ leptons are negligible, the matter potential can be written as

$$
\mathrm{H}_{\text {mat }}=\sqrt{2} G_{\mathrm{F}}\left[\begin{array}{rr}
n_{e} & 0 \\
0 & 0
\end{array}\right]=\frac{\lambda}{2}\left(\mathrm{I}+\sigma_{3}\right),
$$

where $G_{\mathrm{F}}$ is the Fermi constant, $n_{e}$ is the net electron number density, $\lambda=\sqrt{2} G_{\mathrm{F}} n_{e}$, $\mathrm{I}$ is the identity matrix, and $\sigma_{3}$ is the third Pauli matrix. The neutrino(-neutrino coupling) potential in Eq. (4) stems from the coherent forward scattering of the neutrino in question by ambient neutrinos through the neutral-current interaction, 37 and it can be written as

$$
\mathrm{H}_{\nu \nu}=\sqrt{2} G_{\mathrm{F}} \int \frac{\mathrm{d}^{3} p^{\prime}}{(2 \pi)^{3}}\left(1-\hat{\mathbf{v}} \cdot \hat{\mathbf{v}}^{\prime}\right)\left(\rho_{\mathbf{p}^{\prime}}-\bar{\rho}_{\mathbf{p}^{\prime}}\right),
$$

where the physical quantities with primes are for the ambient neutrinos.

We will be interested in the regimes with large matter densities where the (effective) neutrino mixing angle in matter is small and

$$
\mathrm{B}_{\omega}=\mathrm{H}_{\mathrm{vac}}+\mathrm{H}_{\mathrm{mat}} \stackrel{\sqrt{2} G_{\mathrm{F}} n_{e} \gg\left|\Delta m^{2} / 2 E\right|}{\longrightarrow}(\lambda-\eta \omega) \frac{\sigma_{3}}{2},
$$


where $\eta=+1$ and -1 for $\mathrm{NH}$ and $\mathrm{IH}$, respectively, and

$$
\omega(E)=\frac{\left|\Delta m^{2}\right|}{2 E} \cos 2 \theta_{\mathrm{v}} .
$$

We have ignored the trace terms here which have no impact on neutrino oscillations. For antineutrino,

$$
\overline{\mathrm{B}}_{\omega}=-\mathrm{H}_{\mathrm{vac}}+\mathrm{H}_{\mathrm{mat}} \longrightarrow(\lambda+\eta \omega) \frac{\sigma_{3}}{2}=\mathrm{B}_{-\omega} .
$$

Therefore, for the purpose of neutrino oscillations one can treat an antineutrino of oscillation frequency $\omega$ and energy $E$ as a neutrino with oscillation frequency $-\omega$ and energy $-E$. We will adopt this convention throughout this paper.

In the absence of neutrino absorption, emission and collision, it is useful to define (reduced) neutrino flavor (density) matrices

$$
\varrho_{\omega>0, \hat{\mathbf{v}}} \propto \rho_{\mathbf{p}}, \quad \varrho_{\omega<0, \hat{\mathbf{v}}} \propto \bar{\rho}_{\mathbf{p}}
$$

with normalization condition

$$
\operatorname{tr} \varrho_{\omega, \hat{\mathbf{v}}}=1 .
$$

The diagonal elements of $\varrho_{\omega, \hat{v}}$ give the probabilities for the corresponding neutrino $(\omega>0)$ or antineutrino $(\omega<0)$ in the corresponding weak-interaction states. Neutrino flavor matrices $\varrho_{\omega, \hat{\mathbf{v}}}$ obey the e.o.m.

$$
\left(\partial_{t}+\hat{\mathbf{v}} \cdot \nabla\right) \varrho_{\omega, \hat{\mathbf{v}}}=-\mathrm{i}\left[\mathrm{B}_{\omega}+\mathrm{H}_{\nu \nu}, \varrho_{\omega, \hat{\mathbf{v}}}\right] .
$$

\subsection{Bipolar model}

The study of collective neutrino oscillations begins with the bipolar model ${ }^{[17}[19]$ This model describes a homogeneous and isotropic system with $\nu_{e}$ and $\bar{\nu}_{e}$ populations of single energy $E_{0}$ at time $t=0$. The e.o.m. of neutrino oscillations is

$$
\mathrm{i} \partial_{t} \varrho_{\omega}=\left[(\lambda-\eta \omega) \frac{\sigma_{3}}{2}+\mathrm{H}_{\nu \nu}, \varrho_{\omega}\right]
$$

with $\omega= \pm \omega_{0}$, and the neutrino potential is

$$
\mathrm{H}_{\nu \nu}=\mu\left(\varrho_{\omega_{0}}-\alpha \varrho_{-\omega_{0}}\right),
$$

where

$$
\alpha=\frac{n_{\bar{\nu}}}{n_{\nu}}
$$

is the ratio of the density of the antineutrino to that of the neutrino, and

$$
\mu=\sqrt{2} G_{\mathrm{F}} n_{\nu}
$$

is a measure of the strength of the neutrino-neutrino coupling.

If the neutrino density is negligible, Eq. (14) has solution

$$
\varrho_{ \pm \omega_{0}}(t) \approx \exp \left[-\mathrm{i}\left(\lambda \mp \eta \omega_{0}\right) t \frac{\sigma_{3}}{2}\right] \varrho_{ \pm \omega_{0}}(0) \exp \left[\mathrm{i}\left(\lambda \mp \eta \omega_{0}\right) t \frac{\sigma_{3}}{2}\right] .
$$


Because

$$
\varrho_{ \pm \omega_{0}}(0)=\left[\begin{array}{ll}
1 & 0 \\
0 & 0
\end{array}\right]
$$

are diagonal and commute with $\sigma_{3}$, one has

$$
\varrho_{ \pm \omega}(t) \approx\left[\begin{array}{ll}
1 & 0 \\
0 & 0
\end{array}\right] .
$$

In other words, neutrino oscillations are suppressed in the presence of dense matter if there is no ambient neutrino.

However, the presence of dense matter does not necessarily suppress collective oscillations in the dense neutrino medium. This can be seen by performing a "corotating-frame" transformation to the neutrino flavor matrix! 18

$$
\tilde{\varrho}_{\omega}(t)=e^{\mathrm{i} \lambda t \sigma_{3} / 2} \rho_{\omega}(t) e^{-\mathrm{i} \lambda t \sigma_{3} / 2},
$$

which removes the time evolution due to matter. Under this transformation Eq. (14 becomes

$$
\mathrm{i} \partial_{t} \tilde{\varrho}_{\omega}(t)=\left[-\eta \omega \frac{\sigma_{3}}{2}+\tilde{\mathrm{H}}_{\nu \nu}, \tilde{\varrho}_{\omega}\right]
$$

where $\tilde{\mathrm{H}}_{\nu \nu}=\mu\left(\tilde{\varrho}_{\omega_{0}}-\alpha \tilde{\varrho}_{-\omega_{0}}\right)$. Eqs. (14) and $(22)$ are equivalent, and $\varrho$ and $\varrho$ have the same diagonal elements. Therefore, if there exists collective flavor transformation in the bipolar model, a uniform dense matter will not suppress it ${ }^{\mathrm{a}}$ In the rest of the paper we will always work in the corotating frame by taking $\lambda=0$, but we will drop the tilde for simplicity.

To see that a bipolar system can experience collective flavor transformation we note that Eq. 22 is invariant under global phase transformation 41

$$
\varrho_{\omega}(t) \longrightarrow e^{-\mathrm{i} \phi \sigma_{3} / 2} \varrho_{\omega}(t) e^{\mathrm{i} \phi \sigma_{3} / 2},
$$

where $\phi$ is a constant. This symmetry leads to the conservation of the "flavor lepton number (density)" 42

$$
\mathcal{L}=n_{\nu} \operatorname{tr}\left[\left(\varrho_{\omega_{0}}-\alpha \varrho_{-\omega_{0}}\right) \sigma_{3}\right]=\left(n_{\nu_{e}}-n_{\nu_{\tau}}\right)-\left(n_{\bar{\nu}_{e}}-n_{\bar{\nu}_{\tau}}\right) .
$$

The phase symmetry in Eq. 23) also implies the existence of a collective precession solution:

$$
\varrho_{\omega}(t)=e^{-\mathrm{i} \Omega t \sigma_{3} / 2} \varrho_{\omega}(0) e^{\mathrm{i} \Omega t \sigma_{3} / 2},
$$

where $\Omega$ is a common oscillation frequency independent of the oscillation frequency $\omega$ of the individual neutrino.

The bipolar model can be solved analytically. 17 It has also been shown that the bipolar model is equivalent to a gyroscopic pendulum in flavor space, 19 and the collective precession solution in Eq. 25) corresponds to the precession motion of this

${ }^{a}$ However, collective neutrino oscillations can be suppressed by a very large matter density in SNe as shown in Ref. 40 
pendulum. However, this collective precession solution can not be achieved exactly for arbitrary initial conditions just as a gyroscopic pendulum usually experiences both nutation and precession.

The bipolar model is, of course, very different from the neutrino medium in a realistic astrophysical environment. However, it does provide explanations for some of the important results of collective neutrino oscillations in more sophisticated models. For example, one can show that in the collective precession solution neutrinos will remain in the weak-interaction states if the neutrino density is larger than a critical value ${ }^{19}$ [see the discussion in Sec. 2.4 and around Eq. (41)]. This corresponds to the "sleeping-top" regime of a gyroscopic pendulum where the fast spinning top or pendulum defies gravity and spins without wobbling. When the neutrino number density decreases below the critical value, the flavor pendulum begins to wobble about the precession solution. Similar phenomenon was indeed observed in numerical simulations of neutrino oscillations in SNe (see Fig. 1).
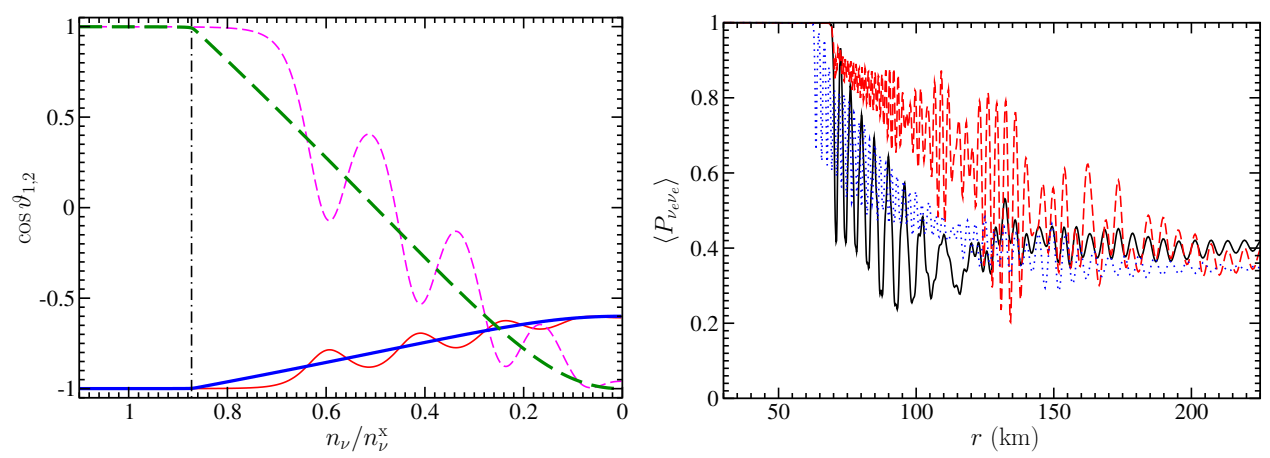

Fig. 1. Left panel: The collective precession solution of a bipolar model with time-varying neutrino density $n_{\nu}$ (thick lines) and the numerical solution for this model (thin lines). The dashed and solid lines represent $\cos \vartheta_{1}=\operatorname{tr}\left(\varrho_{\omega_{0}} \sigma_{3}\right)$ and $\cos \vartheta_{2}=-\operatorname{tr}\left(\varrho_{-\omega_{0}} \sigma_{3}\right)$, respectively. Right panel: The energy-averaged neutrino survival probabilities for three representative neutrino trajectories as functions of radius $r$ in a spherical supernova model. The figures are adapted from Fig. 3 of Ref. 43 and Fig. 2 of Ref. 7 respectively.

\subsection{Spectral swap/split}

The collective precession solution in Eq. 25) also exists in a homogeneous and isotropic neutrino gas with continuous neutrino energy spectra $\stackrel{42}{4}$ We will again assume that the neutrinos and antineutrinos are in the weak-interaction states at time $t=0$. Because Eq. (4) is invariant under $\rho \rightarrow-\rho$ (without changing $\mathrm{H}_{\nu \nu}$ ), we can use

$$
\varrho_{\omega}(t=0)=\left[\begin{array}{ll}
1 & 0 \\
0 & 0
\end{array}\right]
$$


as the initial condition for all neutrinos and antineutrinos by replacing the neutrino energy spectra with a single effective spectrum

$$
g(\omega) \propto\left|\frac{\mathrm{d} E}{\mathrm{~d} \omega}\right| \times \begin{cases}\operatorname{tr}\left(\rho_{E} \sigma_{3}\right)_{t=0} & \text { if } \omega>0 \\ -\operatorname{tr}\left(\bar{\rho}_{E} \sigma_{3}\right)_{t=0} & \text { if } \omega<0\end{cases}
$$

with normalization

$$
\int_{0}^{\infty} g(\omega) \mathrm{d} \omega=1
$$

The e.o.m. (22) also applies to the neutrino gas with continuous neutrino energy spectra except that the neutrino potential is now

$$
\mathrm{H}_{\nu \nu}=\mu \int_{-\infty}^{\infty} g(\omega) \varrho_{\omega} \mathrm{d} \omega,
$$

where

$$
\mu=\sqrt{2} G_{\mathrm{F}}\left(n_{\nu_{e}}-n_{\nu_{\tau}}\right)_{t=0} .
$$

The definition of $\alpha$ in Eq. 16. can be generalized to

$$
\alpha=-\frac{\int_{-\infty}^{0} g(\omega) \mathrm{d} \omega}{\int_{0}^{\infty} g(\omega) \mathrm{d} \omega} .
$$

Obviously, the bipolar model is a special case of the continuous-spectrum model with

$$
g(\omega)=\delta\left(\omega-\omega_{0}\right)-\alpha \delta\left(\omega+\omega_{0}\right) .
$$

If the neutrino gas is in the collective precession mode initially and expands adiabatically afterwards, it should remain in the collective mode. However, according to Eq. 22 , when the neutrino density becomes negligible (at time $t \geq t_{1}$ ), neutrinos of different energies should oscillate independently with their own oscillation frequencies:

$$
\varrho_{\omega}(t)=e^{\mathrm{i} \eta \omega\left(t-t_{1}\right) \sigma_{3} / 2} \varrho_{\omega}\left(t_{1}\right) e^{-\mathrm{i} \eta \omega\left(t-t_{1}\right) \sigma_{3} / 2} .
$$

This apparent contradiction is resolved if $\varrho_{\omega}\left(t_{1}\right)$ is diagonal. Being diagonal does not necessarily mean that $\varrho_{\omega}\left(t_{1}\right)=\varrho_{\omega}(0)=\operatorname{diag}[1,0]$. For example, if the neutrino gas has a positive flavor lepton number $\mathcal{L}$ [see Eq. 224]] and a bipolar-like spectrum [i.e. $g(\omega)$ is positive (negative) if $\omega>0(\omega<0)$ ] and if the neutrino mass hierarchy is inverted, the final spectrum can be split at the critical energy

$$
E_{\mathrm{C}}=\frac{\left|\Delta m^{2}\right|}{2 \Omega_{0}}
$$

\footnotetext{
${ }^{\mathrm{b}}$ Other equivalent normalization conditions also exist in the literature. The normalization condition in Eq. $(28)$ is used in anticipation that the $\nu_{e}$ flux is the largest among all flavors in a SN.
} 
such that

$$
\varrho_{\omega}\left(t_{1}\right)= \begin{cases}\operatorname{diag}[1,0] & \text { if } \omega>\Omega_{0}, \\ \operatorname{diag}[0,1] & \text { if } \omega<\Omega_{0},\end{cases}
$$

where $\Omega_{0}$ is the collective oscillation frequency at $\mu=0^{6}$ and can be determined by the conservation of $\mathcal{L} 42$ The above result implies that $\nu_{e}$ and $\nu_{\tau}$ have swapped their energy spectra at energies $E>E_{\mathrm{C}}$. This spectral swap/split phenomenon is commonly observed in the numerical simulations of neutrino oscillations in $\mathrm{SNe}$ (see Fig. 22). If the neutrino gas has a more complicated spectra with multiple spectral crossings where $g(\omega)=0$, the final spectra can exhibit multiple spectral splits. $\frac{10}{10}$
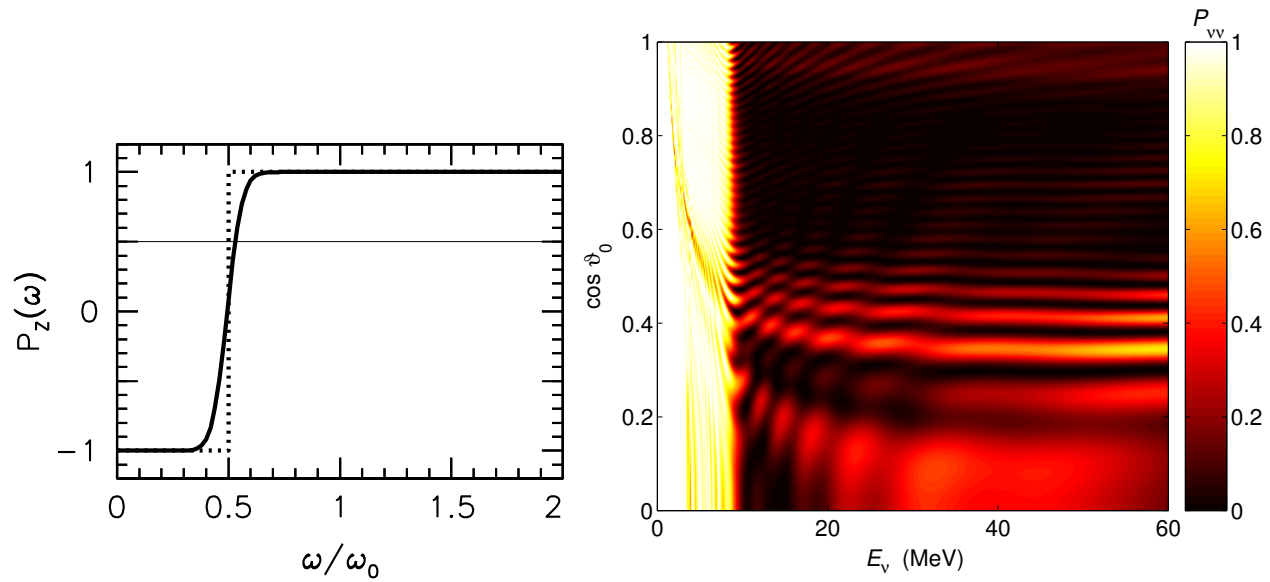

Fig. 2. Left panel: The split neutrino spectrum (dotted line: fully adiabatic; thick solid line: numerical) produced from a box-like initial spectrum (thin solid line) in a homogeneous and isotropic neutrino gas by collective neutrino oscillations, where $\omega=\left|\Delta m^{2}\right| / 2 E_{\nu}$ and $P_{z}(\omega)=$ $\operatorname{tr}\left(\varrho_{\omega}\right)$. Right panel: The final neutrino survival probability as a function of neutrino energy $E_{\nu}$ and neutrino emission angle $\vartheta_{0}$ on the surface of the proto-neutron star in a numerical calculation employing a spherical supernova model. The figures are adapted from Fig. 1 of Ref. 42 and Fig. 3 of Ref. 7], respectively.

\subsection{Flavor instability}

Although the bipolar model can be solved analytically and provide some qualitative understandings of collective oscillations in neutrino media, numerical computations are usually required to obtain quantitative results of neutrino oscillations in more sophisticated models. However, if the neutrinos and antineutrinos are initially in the weak-interaction states, linear stability analysis can be utilized to predict the physical regimes where collective oscillations can occur without performing largescale numerical simulations. This is especially useful if a large parameter space needs to be surveyed because of the uncertainty in, e.g., the neutrino fluxes in 
SN simulations. The linear stability analysis method was explained thoroughly in Ref. 20. Here we will use the bipolar model to demonstrate the essence of this method.

We assume that no significant flavor oscillations have occurred at time $t$ such that

$$
\varrho_{\omega_{0}} \approx\left[\begin{array}{cc}
1 & \epsilon \\
\epsilon^{*} & 0
\end{array}\right], \quad \varrho_{-\omega_{0}} \approx\left[\begin{array}{cc}
1 & \bar{\epsilon} \\
\bar{\epsilon}^{*} & 0
\end{array}\right],
$$

where $|\epsilon| \sim|\bar{\epsilon}| \ll 1$. Keeping only the terms up to $\mathcal{O}(\epsilon)$ we can rewrite Eq. (22) in terms of $\epsilon$ and $\bar{\epsilon}$ as

$$
\mathrm{i} \partial_{t}\left[\begin{array}{l}
\epsilon \\
\bar{\epsilon}
\end{array}\right] \approx\left[\begin{array}{cc}
-\eta \omega-\mu \alpha & \alpha \mu \\
-\mu & \eta \omega+\mu
\end{array}\right]\left[\begin{array}{l}
\epsilon \\
\bar{\epsilon}
\end{array}\right]=\Lambda \cdot\left[\begin{array}{l}
\epsilon \\
\bar{\epsilon}
\end{array}\right] .
$$

Note that, in the absence of the neutrino medium (i.e. $\mu=0$ ), $\epsilon$ and $\bar{\epsilon}$ are decoupled and oscillate with frequencies $-\eta \omega$ and $\eta \omega$, respectively. In the neutrino medium, however, $\epsilon$ and $\bar{\epsilon}$ are coupled, and each eigenvalue $\Omega$ of matrix $\Lambda$ corresponds to a collective oscillation mode:

$$
\epsilon(t) \approx Q e^{-\mathrm{i} \Omega t}, \quad \bar{\epsilon}(t) \approx \bar{Q} e^{-\mathrm{i} \Omega t},
$$

where $[Q, \bar{Q}]^{T}$ is the corresponding eigenvector of $\Lambda$.

If $\Omega$ is real, $\epsilon$ and $\bar{\epsilon}$ will oscillate and their amplitudes will remain small. If $\Omega$ is complex with

$$
\kappa=\operatorname{Im}(\Omega)>0,
$$

the bipolar model has a flavor instability: the amplitudes of $\epsilon$ and $\bar{\epsilon}$ will grow exponentially which can lead to (large-magnitude) collective oscillations. Because $\Lambda$ is a real matrix, when $\Omega$ is complex, $\Omega^{*}$ is also an eigenvalue of $\Lambda$. In this case, the solution with positive $\kappa$ will dominate over time. Therefore, for the purpose of flavor stability analysis, it is sufficient to find out the regimes where $\Lambda$ has complex eigenvalues.

It is straightforward to show that $\Lambda$ has complex eigenvalues only when

$$
(1-\alpha)^{2} \mu^{2}+4 \omega_{0}^{2}+4(1+\alpha) \mu \eta \omega_{0}<0
$$

or

$$
\frac{2 \omega_{0}}{(1+\sqrt{\alpha})^{2}}<-\eta \mu<\frac{2 \omega_{0}}{(1-\sqrt{\alpha})^{2}} .
$$

The bipolar model (with $\nu_{e}$ and $\bar{\nu}_{e}$ initially) can have flavor instability only in IH. The upper limit of $\mu$ where collective oscillations can occur indeed agrees with the result plotted in Fig. 1(a). It is interesting to note that, if the bipolar model has $\nu_{\tau}$ and $\bar{\nu}_{\tau}$ initially instead of $\nu_{e}$ and $\bar{\nu}_{e}$, it will have flavor instability only in $\mathrm{NH}$ because $\mu<0$ [see Eq. (30)].

Similarly, for the continuous-spectrum model, we assume

$$
\varrho_{\omega} \approx\left[\begin{array}{cc}
1 & \epsilon_{\omega} \\
\epsilon_{\omega}^{*} & 0
\end{array}\right],
$$


the e.o.m. 222 can be written as

$$
\mathrm{i} \partial_{t} \epsilon_{\omega} \approx[-\eta \omega+(1-\alpha) \mu] \epsilon_{\omega}-\mu \int_{-\infty}^{\infty} g\left(\omega^{\prime}\right) \epsilon_{\omega^{\prime}} \mathrm{d} \omega^{\prime} .
$$

Using the ansatz

$$
\epsilon_{\omega}(t)=Q_{\omega} e^{-\mathrm{i} \Omega t}
$$

one obtains

$$
[\Omega-(1-\alpha) \mu+\eta \omega] Q_{\omega}=\text { const. } \quad \text { or } \quad Q_{\omega} \propto \frac{1}{\Omega-(1-\alpha) \mu+\eta \omega} .
$$

In order for the above solution be consistent with Eq. (43), $\Omega$ must satisfy the consistency condition

$$
-\frac{1}{\mu}=\int_{-\infty}^{\infty} \frac{g(\omega)}{\Omega-(1-\alpha) \mu+\eta \omega} \mathrm{d} \omega
$$

Because $\widetilde{\Omega}=\Omega-(1-\alpha) \mu$ and $\Omega$ have the same imaginary part, it is sufficient to solve

$$
-\frac{1}{\mu}=\int_{-\infty}^{\infty} \frac{g(\omega)}{\widetilde{\Omega}+\eta \omega} \mathrm{d} \omega
$$

for $\widetilde{\Omega}$ for the purpose of flavor stability analysis. A complex $\widetilde{\Omega}$ implies the existence of a flavor instability.

Flavor stability analysis can also be applied to more complicated models. Although a powerful tool, the linear stability analysis method depends on the validity of assumption (42) and is limited to the linear regime. When there is a flavor instability, numerical simulations are still needed to follow neutrino oscillations in the nonlinear regime.

\section{Spontaneous symmetry breaking}

The e.o.m. 133 involves seven dimensions and has not been solved in its complete form. Instead, various symmetries have been assumed to reduce the dimensionality of the problem so that a numerical or analytically solution can be found. Three classes of symmetries are commonly employed: the time translation symmetry that makes the problem time-independent, spatial symmetries that reduce the spatial dimensions, and directional symmetries that reduce the momentum dimensions. For example, a commonly used model for the early universe assumes complete (spatial) homogeneity and (directional) isotropy $\sqrt[3]{44}$ These assumptions reduce the neutrino transport in the early universe to a two-dimensional problem. Another example is the $\mathrm{SN}$ neutrino Bulb mode 6 which assumes the time translation symmetry, the (spatial) spherical symmetry about the center of the SN, and the (directional) axial symmetry about the radial direction. These assumptions reduce the neutrino transport in the SN to a three-dimension problem. 
However, the full e.o.m. (13) allows solutions that may or may not have these symmetries. If a symmetry-breaking solution becomes unstable, collective oscillations can break the corresponding symmetry spontaneously even if such an (approximate) symmetry exists in the neutrino medium initially ${ }^{21}$ We will first use the two-beam model to illustrate the basic idea of spontaneous symmetry breaking by collective oscillations. We will then look at the breaking of directional and spatial symmetries separately.

\subsection{Two-beam model}

We consider a model in which all neutrinos are emitted from the $x$ axis and propagate only in the $x-z$ plane. We assume that neutrinos are emitted in only two directions:

$$
\hat{\mathbf{v}}_{\zeta}=\left[u_{\zeta}, 0, v_{z}\right] \quad(\zeta=L, R),
$$

where $0<v_{z}<1$ and $u_{R}=-u_{L}=\sqrt{1-v_{z}^{2}}$. We also assume that every point on the $x$ axis emits in each direction neutrinos and antineutrinos of single energy $E_{0}$ with intensities $j_{\nu}$ and $j_{\bar{\nu}}=\alpha j_{\nu}$, respectively. We further impose the time translation symmetry and the translation symmetry along the $x$ axis. With these conditions Eq. (13) reduces to

$$
\mathrm{i} \hat{\mathbf{v}}_{\zeta} \cdot \nabla \varrho_{\omega, \zeta}=\left[-\eta \omega \frac{\sigma_{3}}{2}+\mathrm{H}_{\nu \nu}, \varrho_{\omega, \zeta}\right] .
$$

In the above equation the neutrino potential is

$$
\begin{aligned}
\mathrm{H}_{\nu \nu} & =\sqrt{2} G_{\mathrm{F}} j_{\nu} \sum_{\zeta^{\prime}}\left(1-\hat{\mathbf{v}}_{\zeta} \cdot \hat{\mathbf{v}}_{\zeta^{\prime}}\right) \int_{-\infty}^{\infty} g\left(\omega^{\prime}\right) \varrho_{\omega^{\prime}, \zeta^{\prime}} \mathrm{d} \omega^{\prime} \\
& =\mu\left(\varrho_{\omega_{0}, \tilde{\zeta}}-\alpha \varrho_{-\omega_{0}, \tilde{\zeta}}\right),
\end{aligned}
$$

where

$$
\mu=\sqrt{2}\left(1-\hat{\mathbf{v}}_{L} \cdot \hat{\mathbf{v}}_{R}\right) G_{\mathrm{F}} j_{\nu},
$$

and $\tilde{\zeta}=R, L$ are the opposites of $\zeta$.

The two beam model has been solved analytically ${ }^{23}$ Here we use the linear stability analysis method to demonstrate that the left-right symmetry $(L \leftrightarrow R)$ can be broken spontaneously. ${ }^{24}$ We again assume that the neutrinos and antineutrinos are in the (almost pure) electron flavor:

$$
\varrho_{\omega_{0}, \zeta} \approx\left[\begin{array}{cc}
1 & \epsilon_{\zeta} \\
\epsilon_{\zeta}^{*} & 0
\end{array}\right], \quad \varrho_{-\omega_{0}, \zeta} \approx\left[\begin{array}{cc}
1 & \bar{\epsilon}_{\zeta} \\
\bar{\epsilon}_{\zeta}^{*} & 0
\end{array}\right] .
$$

We then define

$$
\epsilon^{ \pm}=\frac{\epsilon_{L} \pm \epsilon_{R}}{\sqrt{2}}, \quad \quad \bar{\epsilon}^{ \pm}=\frac{\bar{\epsilon}_{L} \pm \bar{\epsilon}_{R}}{\sqrt{2}} .
$$


Keeping only the terms up to $\mathcal{O}(\epsilon)$ we can rewrite Eq. (49) as

$$
\mathrm{i} \partial_{z}\left[\begin{array}{l}
\epsilon^{+} \\
\bar{\epsilon}^{+} \\
\epsilon^{-} \\
\bar{\epsilon}^{-}
\end{array}\right] \approx\left[\begin{array}{lll}
\Lambda_{+} & \\
& \Lambda_{-}
\end{array}\right] \cdot\left[\begin{array}{l}
\epsilon^{+} \\
\bar{\epsilon}^{+} \\
\epsilon^{-} \\
\bar{\epsilon}^{-}
\end{array}\right],
$$

where

$$
\begin{aligned}
& \Lambda_{+}=v_{z}^{-1}\left[\begin{array}{cc}
-\eta \omega_{0}-\alpha \mu & \alpha \mu \\
-\mu & \eta \omega_{0}+\mu
\end{array}\right], \\
& \Lambda_{-}=v_{z}^{-1}\left[\begin{array}{cc}
-\eta \omega_{0}+(2-\alpha) \mu & -\alpha \mu \\
\mu & \eta \omega_{0}+(1-2 \alpha) \mu
\end{array}\right] .
\end{aligned}
$$

Therefore, the plus modes $\left[\epsilon^{+}, \bar{\epsilon}^{+}\right]$and the minus modes $\left[\epsilon^{-}, \bar{\epsilon}^{-}\right]$evolve independently in the linear regime.

The two-beam model is a 3-dimensional model with 1 spatial dimension $(z)$ and 2 momentum dimensions $(\omega, \zeta)$. Its e.o.m. $(49)$ is invariant under the simultaneous interchange of the left and right neutrino beams. When this left-right symmetry is artificially imposed, Eq. 49 reduces to the e.o.m. of the 2-dimensional bipolar model [Eq. 222]]. The bipolar model permits only the symmetry-preserving solutions which can be unstable only in IH (see Sec. 2.4). However, because

$$
\Lambda_{-}=-\left.\Lambda_{+}\right|_{\eta \rightarrow-\eta}+2(1-\alpha) \mu \mathrm{l},
$$

the minus modes in the two-beam model are unstable in the same range of $\mu$ in $\mathrm{NH}$ as the plus modes in IH. In the regime where the minus modes become unstable, the left-right symmetry in the two-beam model is broken spontaneously, and the neutrino oscillations in the two-beam model and the bipolar model are qualitatively different.

\subsection{Breaking of the directional symmetry}

The breaking of the left-right symmetry in the two-beam model is an example of how the directional symmetry can be broken during neutrino oscillations. The origin of this symmetry breaking lies in the current-current nature of the weak coupling. This is best illustrated using the bipolar model in Sec. 2.2 but without imposing the isotropic condition. ${ }^{24}$ The e.o.m. of this model is

$$
\mathrm{i} \partial_{t} \varrho_{\omega, \hat{\mathbf{v}}}=\left[-\eta \omega \frac{\sigma_{3}}{2}+\mathrm{H}_{\nu \nu}, \varrho_{\omega, \hat{\mathbf{v}}}\right],
$$

where

$$
\mathbf{H}_{\nu \nu}=\frac{\sqrt{2}}{4 \pi} G_{\mathrm{F}} n_{\nu} \int \mathrm{d} \Gamma_{\hat{\mathbf{v}}^{\prime}}\left(1-\hat{\mathbf{v}} \cdot \hat{\mathbf{v}}^{\prime}\right)\left(\varrho_{\omega_{0}, \hat{\mathbf{v}}^{\prime}}-\alpha \varrho_{-\omega_{0}, \hat{\mathbf{v}}^{\prime}}\right)
$$

with $\mathrm{d} \Gamma_{\hat{\mathbf{v}}^{\prime}}$ being the differential solid angle around direction $\hat{\mathbf{v}}^{\prime}$. We will again assume that

$$
\varrho_{\omega_{0}, \hat{\mathbf{v}}} \approx\left[\begin{array}{cc}
1 & \epsilon_{\hat{\mathbf{v}}} \\
\epsilon_{\hat{\mathbf{v}}}^{*} & 0
\end{array}\right], \quad \varrho_{-\omega_{0}, \hat{\mathbf{v}}} \approx\left[\begin{array}{cc}
1 & \bar{\epsilon}_{\hat{\mathbf{v}}} \\
\bar{\epsilon}_{\hat{\mathbf{v}}}^{*} & 0
\end{array}\right]
$$


We now switch to the basis of spherical harmonics $Y_{l, m}(\hat{\mathbf{v}})$ and define

$$
\epsilon_{l, m}=\int Y_{l, m}^{*}(\hat{\mathbf{v}}) \epsilon_{\hat{\mathbf{v}}} \mathrm{d} \Gamma_{\hat{\mathbf{v}}}, \quad \quad \bar{\epsilon}_{l, m}=\int Y_{l, m}^{*}(\hat{\mathbf{v}}) \bar{\epsilon}_{\hat{\mathbf{v}}} \mathrm{d} \Gamma_{\hat{\mathbf{v}}} .
$$

Using identity

$$
1-\hat{\mathbf{v}} \cdot \hat{\mathbf{v}}^{\prime}=4 \pi\left[Y_{0,0}(\hat{\mathbf{v}}) Y_{0,0}^{*}\left(\hat{\mathbf{v}}^{\prime}\right)-\frac{1}{3} \sum_{m=0, \pm 1} Y_{1, m}(\hat{\mathbf{v}}) Y_{1, m}^{*}\left(\hat{\mathbf{v}}^{\prime}\right)\right]
$$

one can rewrite Eq. (57) in the linear regime and in the spherical basis as

$$
\mathrm{i} \partial_{t}\left[\begin{array}{c}
\epsilon_{l, m} \\
\bar{\epsilon}_{l, m}
\end{array}\right] \approx \Lambda_{l} \cdot\left[\begin{array}{c}
\epsilon_{l, m} \\
\bar{\epsilon}_{l, m}
\end{array}\right]
$$

where

$$
\Lambda_{l}=(1-\alpha) \mu_{0} I+\left[\begin{array}{cc}
-\eta \omega_{0}-\mu_{l} & \alpha \mu_{l} \\
-\mu_{l} & \eta \omega_{0}+\alpha \mu_{l}
\end{array}\right]
$$

with

$$
\mu_{l}= \begin{cases}\sqrt{2} G_{\mathrm{F}} n_{\nu} & \text { if } l=0 \\ -(\sqrt{2} / 3) G_{\mathrm{F}} n_{\nu} & \text { if } l=1, \\ 0 & \text { otherwise. }\end{cases}
$$

We note that the coupling coefficients $\mu_{l}$ of the monopole mode $(l=0)$ and the dipole modes $(l=1)$ have opposite signs. This sign difference is derived from the current-current nature of the weak coupling which is proportional to $\left(1-\hat{\mathbf{v}} \cdot \hat{\mathbf{v}}^{\prime}\right)$ [see Eq. 61]].

The homogeneous neutrino gas model is 4 dimensional with 1 temporal dimensions and 3 momentum dimensions. Its e.o.m. (57) is invariant under a simultaneous but arbitrary rotation of the momenta of all the neutrinos and antineutrinos. When this rotation symmetry in momentum space is artificially imposed, Eq. (57) reduces to the e.o.m. of the 2-dimensional bipolar model. The bipolar model permits only the symmetric solutions which have flavor instabilities only in IH. However, because $\mu_{l=1}<0$, the dipole modes in the homogeneous gas model have flavor instabilities in $\mathrm{NH}$ [see the discussion around Eq. [41)] which is clearly shown in Fig. 3 . In the regime where the dipole modes become unstable, the rotation symmetry in the homogeneous gas model is broken spontaneously which makes it qualitatively different from the bipolar model.

The above result can also be extended to SN models. As mentioned previously, a commonly used SN model in collective neutrino oscillations is the Bulb model which has the (spatial) spherical symmetry about the center of the SN and the (directional) axial symmetry about the radial direction. We define moment basis function

$$
\Phi_{m}(\varphi)=\frac{e^{\mathrm{i} \varphi}}{\sqrt{2 \pi}} \quad(m=0, \pm 1, \ldots),
$$



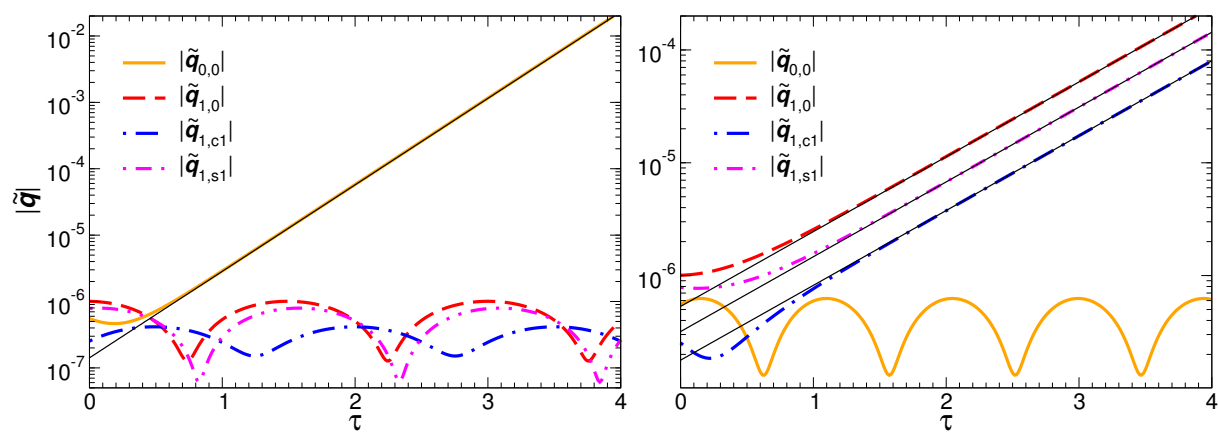

Fig. 3. The exponential growth of the amplitude of the monopole mode $\left(\left|\tilde{\mathbf{q}}_{0,0}\right| \propto\left|\epsilon_{0,0}\right|\right)$ in the inverted neutrino mass hierarchy (left) and the dipole modes $\left(\left|\tilde{\mathbf{q}}_{1,0}\right| \propto\left|\epsilon_{1,0}\right|,\left|\tilde{\mathbf{q}}_{1, s 1}\right| \propto \mid\left(\epsilon_{1,1}-\right.\right.$ $\left.\epsilon_{1,-1}\right) / 2 \mathrm{i} \mid$ and $\left.\left|\tilde{\mathbf{q}}_{1, c 1}\right| \propto\left|\left(\epsilon_{1,1}+\epsilon_{1,-1}\right) / 2\right|\right)$ in the normal neutrino mass hierarchy (right) in a homogeneous neutrino gas model. The figure is adapted from Fig. 1 of Ref. 24

where $\varphi$ is the azimuthal angle of $\hat{\mathbf{v}}$ about the radial direction. Using identity

$$
1-\hat{\mathbf{v}} \cdot \hat{\mathbf{v}}^{\prime}=2 \pi\left[\left(1-\cos \vartheta \cos \vartheta^{\prime}\right) \Phi_{0}(\varphi) \Phi_{0}^{*}\left(\varphi^{\prime}\right)-\frac{1}{2} \sin \vartheta \sin \vartheta^{\prime} \sum_{m= \pm 1} \Phi_{m}(\varphi) \Phi_{m}^{*}\left(\varphi^{\prime}\right)\right]
$$

one sees that the coupling coefficients of the $|m|=0$ and 1 modes have opposite signs, where $\vartheta$ is the angle that $\hat{\mathbf{v}}$ makes with the radial direction. Similar to the homogeneous neutrino gas mdoel, only the $m=0$ modes can exist in the Bulb model because of its imposed axial symmetry. As a result, collective neutrino oscillations occur only in IH if the neutrino fluxes are bipolar-like. If the axial symmetry is not imposed, however, collective oscillations can also occur in $\mathrm{NH}$ through the collective modes with $m= \pm 1$ which break the axial symmetry spontaneously. (See Ref. 21 for a detailed analysis and Ref. 22 for the numerical confirmation of this analysis.)

\subsection{Breaking of the spatial symmetry}

Spatial symmetries can also be broken by collective neutrino oscillations. This can be illustrated by using the (neutrino) Line mode ${ }^{26}$ which is a generalization of the two-beam model in Sec. 3.1. Compared to the two-beam model, the Line model does not possess the translation symmetry along the $x$ axis. But for computational purpose we will impose a periodic condition

$$
\varrho_{\omega, \zeta}(x, z)=\varrho_{\omega, \zeta}(x+L, z),
$$

where $L$ is constant. We will again assume that neutrinos and antineutrinos are in the (almost pure) electron flavor [see Eqs. 52 and $[53 \mathrm{~B}]$. It is convenient to work in the Fourier basis and define

$$
\epsilon_{m}^{ \pm}(z)=\frac{1}{L} \int_{0}^{L} e^{-\mathrm{i} m k_{0} x} \epsilon^{ \pm}(x, z) \mathrm{d} x, \quad \bar{\epsilon}_{m}^{ \pm}(z)=\frac{1}{L} \int_{0}^{L} e^{-\mathrm{i} m k_{0} x} \bar{\epsilon}^{ \pm}(x, z) \mathrm{d} x,
$$


where $k_{0}=2 \pi / L$. In the linear regime the Fourier modes with different $m$ values are decoupled, and the linearized e.o.m. are

$$
\mathrm{i} \partial_{z}\left[\begin{array}{c}
\epsilon_{m}^{+} \\
\bar{\epsilon}_{m}^{+} \\
\epsilon_{m}^{-} \\
\bar{\epsilon}_{m}^{-}
\end{array}\right] \approx \Lambda_{m} \cdot\left[\begin{array}{c}
\epsilon_{m}^{+} \\
\bar{\epsilon}_{m}^{+} \\
\epsilon_{m}^{-} \\
\bar{\epsilon}_{m}^{-}
\end{array}\right]
$$

where

$$
\Lambda_{m}=v_{z}^{-1}\left[\begin{array}{cccc}
-\eta \omega_{0}-\alpha \mu & \alpha \mu & m q & 0 \\
-\mu & \eta \omega_{0}+\mu & 0 & m q \\
m q & 0 & -\eta \omega_{0}+(2-\alpha) \mu & -\alpha \mu \\
0 & m q & \mu & \eta \omega_{0}+(1-2 \alpha) \mu
\end{array}\right]
$$

with $q=(2 \pi / L) \sqrt{1-v_{z}^{2}}$.

The Line model is a 4 -dimensional model with 2 spatial dimensions $(x, z)$ and 2 momentum dimensions $(\omega, \zeta)$. Its e.o.m. is invariant under the translation symmetry along the $x$ direction. When this symmetry is artificially imposed, the Line model reduces to the 3-dimensional two-beam model where only the homogeneous modes (with $m=0$ ) are allowed. The Line model allows both the homogeneous and inhomogeneous $(m \neq 0)$ modes, and the latter break the translation symmetry spontaneously when they become unstable.

For given values of $\mu$ and $\alpha$ one can find out if there is a flavor instability by computing the eigenvalues of $\Lambda$. It turns out that the (two-beam) Line model has the same flavor unstable regions [in terms of $(\mu, m)$ ] for both IH and NH. Fig. 4 shows the flavor unstable region in the $(\mu, m)$ space for the case with $\alpha=0.8$ and $L=20 \pi / \omega_{0}$. Note that the inhomogeneous modes with large $|m|$ values occur on small distance scales, and they can be unstable at larger neutrino densities than the homogeneous mode does.

\section{Summary and outlook}

Neutrino flavor transport in hot and dense astrophysical environments is a difficult seven-dimensional nonlinear problem. Using the simplest neutrino gas model, i.e. the bipolar model, we reviewed some of the key concepts of collective neutrino oscillations. By applying the method of linear stability analysis we illustrated that how the directional and spatial symmetries may be broken spontaneously during collective neutrino oscillations.

The realization of the spontaneous symmetry breaking commences a new stage of the study collective neutrino oscillations. All the previous research is limited to the models with imposed symmetries which have smaller dimensions than real physical systems do. The studies of these simplified models have yielded important insights into the intriguing phenomena of collective neutrino oscillations, and some of the toy models shall still prove useful in understanding the results in more realistic 


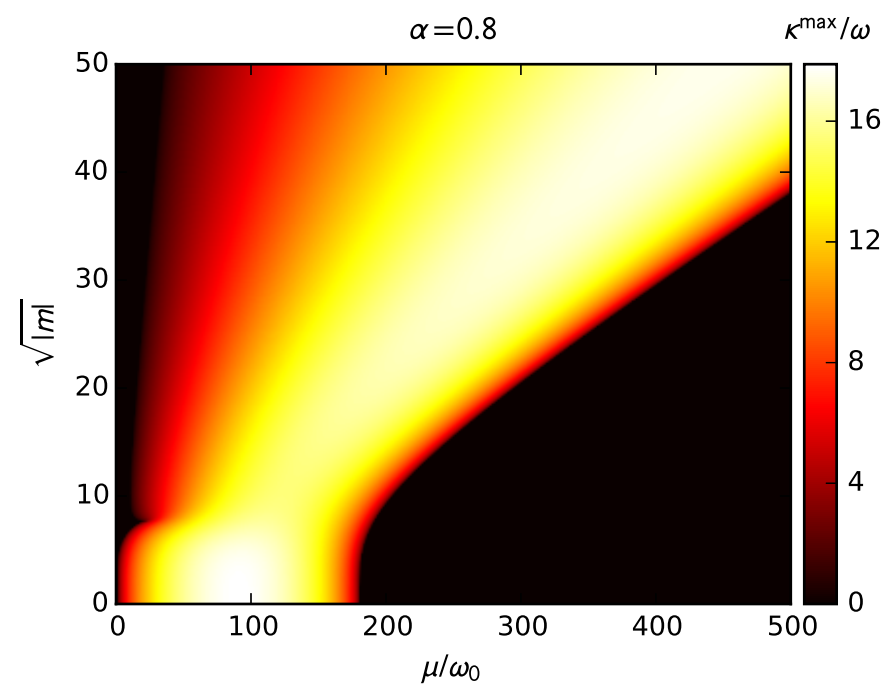

Fig. 4. The flavor instabilities of the Line model with $\alpha=0.8$. The color scale represents $\kappa_{m}^{\max }(\mu)$, the largest exponential growth rate of the collective oscillation modes. The size of the periodic box along the $x$-axis is taken to be $L=20 \pi / \omega_{0}$, and the propagation directions of the neutrinos are given by unit vectors $\left[v_{x}, v_{z}\right]=[ \pm \sqrt{3} / 2,1 / 2]$. The results are independent of the neutrino mass hierarchy. The figure is adapted from Fig. 1 of Ref. 26

models. However, the incompatibility of the reduced-dimension models and collective neutrino oscillations clearly points out that these models are insufficient and that dimensionality really matters in collective oscillations. For example, a previous comparison between the results obtained using the single-angle and multi-angle SN models shows that neutrino oscillations in these two models of different dimensions can have completely different yields of heavy elements in SN neutrino-driven wind $\stackrel{45}{5}$ The results of the Line model suggest that neutrino oscillations can occur at larger neutrino densities in the models of multiple spatial dimensions than in the corresponding models with only one spatial dimension. Although a very challenging task, new and realistic multi-dimensional neutrino gas models must be constructed and employed before we can know with any confidence what true physical impact neutrino oscillations may have on astrophysical environments with dense neutrino media such $\mathrm{SNe}$ and black-hole accretion discs.

\section{Acknowledgements}

This work was supported by DOE EPSCoR grant de-sc0008142 at UNM. We thank S. Abbar and S. Shalgar for useful discussions. We also appreciate the hospitality of INT/UW where part of this work was finished. 


\section{References}

1. S. Woosley and T. Janka, Nature Physics 1 (2005) 147.

2. Particle Data Group Collaboration (K. Olive et al.), Chin.Phys. C38 (2014) 090001.

3. V. A. Kostelecky and S. Samuel, Phys.Lett. B318 (1993) 127.

4. S. Pastor and G. Raffelt, Phys.Rev.Lett. 89 (2002) 191101, arXiv:astro-ph/0207281 [astro-ph]

5. A. Balantekin and H. Yuksel, New J.Phys. 7 (2005) 51, arXiv:astro-ph/0411159 [astro-ph]

6. H. Duan, G. M. Fuller, J. Carlson and Y.-Z. Qian, Phys.Rev. D74 (2006) 105014, arXiv:astro-ph/0606616 [astro-ph].

7. H. Duan, G. M. Fuller, J. Carlson and Y.-Z. Qian, Phys.Rev.Lett. 97 (2006) 241101, arXiv:astro-ph/0608050 [astro-ph].

8. G. L. Fogli, E. Lisi, A. Marrone and A. Mirizzi, JCAP 0712 (2007) 010, arXiv:0707.1998 [hep-ph].

9. H. Duan, G. M. Fuller, J. Carlson and Y.-Z. Qian, Phys.Rev.Lett. 100 (2008) 021101, arXiv:0710.1271 [astro-ph]

10. B. Dasgupta, A. Dighe, G. G. Raffelt and A. Y. Smirnov, Phys.Rev.Lett. 103 (2009) 051105, arXiv:0904.3542 [hep-ph]

11. A. Friedland, Phys.Rev.Lett. 104 (2010) 191102, arXiv:1001.0996 [hep-ph].

12. A. Mirizzi and P. D. Serpico, Phys.Rev.Lett. 108 (2012) 231102, arXiv:1110.0022 [hep-ph]

13. S. Galais, J. Kneller and C. Volpe, J.Phys. G39 (2012) 035201, arXiv:1102.1471 [astro-ph.SR].

14. J. F. Cherry, J. Carlson, A. Friedland, G. M. Fuller and A. Vlasenko, Phys.Rev.Lett. 108 (2012) 261104, arXiv:1203.1607 [hep-ph]

15. A. de Gouvea and S. Shalgar, JCAP 1210 (2012) 027, arXiv:1207.0516 [astro-ph.HE].

16. A. Malkus, A. Friedland and G. McLaughlin (2014) arXiv:1403.5797 [hep-ph]

17. V. A. Kostelecky and S. Samuel, Phys.Rev. D52 (1995) 621, arXiv:hep-ph/9506262 [hep-ph]

18. H. Duan, G. M. Fuller and Y.-Z. Qian, Phys.Rev. D74 (2006) 123004, arXiv:astro-ph/0511275 [astro-ph].

19. S. Hannestad, G. G. Raffelt, G. Sigl and Y. Y. Wong, Phys.Rev. D74 (2006) 105010, arXiv:astro-ph/0608695 [astro-ph].

20. A. Banerjee, A. Dighe and G. Raffelt, Phys.Rev. D84 (2011) 053013, arXiv:1107.2308 [hep-ph]

21. G. Raffelt, S. Sarikas and D. de Sousa Seixas, Phys.Rev.Lett. 111 (2013) 091101, arXiv:1305.7140 [hep-ph].

22. A. Mirizzi, Phys.Rev. D88 (2013) 073004, arXiv:1308.1402 [hep-ph]

23. G. Raffelt and D. d. S. Seixas, Phys. Rev., D88 (2013) 045031, arXiv:1307.7625 [hep-ph]

24. H. Duan, Phys.Rev. D88 (2013) 125008, arXiv:1309.7377 [hep-ph]

25. G. Mangano, A. Mirizzi and N. Saviano, Phys.Rev. D89 (2014) 073017, arXiv:1403.1892 [hep-ph].

26. H. Duan and S. Shalgar, Phys.Lett. B747 (2015) 139, arXiv:1412.7097 [hep-ph]

27. A. Mirizzi, G. Mangano and N. Saviano (2015) arXiv:1503.03485 [hep-ph]

28. B. Dasgupta and A. Dighe, Phys.Rev. D77 (2008) 113002, arXiv:0712.3798 [hep-ph]

29. H. Duan, G. M. Fuller and Y.-Z. Qian, Phys.Rev. D77 (2008) 085016, arXiv:0801.1363 [hep-ph]. 
30. H. Duan, G. M. Fuller and Y.-Z. Qian, Ann.Rev.Nucl.Part.Sci. 60 (2010) 569, arXiv:1001.2799 [hep-ph].

31. A. Friedland and C. Lunardini, JHEP 0310 (2003) 043, arXiv:hep-ph/0307140 [hep-ph]

32. Y. Pehlivan, A. Balantekin, T. Kajino and T. Yoshida, Phys.Rev. D84 (2011) 065008 , arXiv: 1105.1182 [astro-ph.CO]

33. G. Sigl and G. Raffelt, Nucl.Phys. B406 (1993) 423.

34. P. Strack and A. Burrows, Phys.Rev. D71 (2005) 093004, arXiv:hep-ph/0504035 [hep-ph]

35. C. Y. Cardall, Phys.Rev. D78 (2008) 085017, arXiv:0712.1188 [astro-ph]

36. L. Wolfenstein, Phys.Rev. D17 (1978) 2369.

37. G. M. Fuller, R. W. Mayle, J. R. Wilson and D. N. Schramm, Astrophys. J. 322 (1987) 795 .

38. D. Notzold and G. Raffelt, Nucl.Phys. B307 (1988) 924.

39. J. T. Pantaleone, Phys.Rev. D46 (1992) 510.

40. A. Esteban-Pretel, A. Mirizzi, S. Pastor, R. Tomas, G. Raffelt et al., Phys.Rev. D78 (2008) 085012, arXiv:0807.0659 [astro-ph]

41. H. Duan, G. M. Fuller and Y.-Z. Qian, J.Phys. G36 (2009) 105003, arXiv:0808.2046 [astro-ph]

42. G. G. Raffelt and A. Y. Smirnov, Phys.Rev. D76 (2007) 081301, arXiv:0705.1830 [hep-ph]

43. H. Duan, G. M. Fuller, J. Carlson and Y.-Z. Qian, Phys.Rev. D75 (2007) 125005, arXiv:astro-ph/0703776 [astro-ph].

44. K. N. Abazajian, J. F. Beacom and N. F. Bell, Phys.Rev. D66 (2002) 013008, arXiv:astro-ph/0203442 [astro-ph].

45. H. Duan, A. Friedland, G. C. McLaughlin and R. Surman, J. Phys. G38 (2011) 035201, arXiv: 1012.0532 [astro-ph.SR]. 